\title{
Correction to: Preventing tuberculosis in paediatric kidney transplant recipients: is there a role for BCG immunisation pre-transplantation in low tuberculosis incidence countries?
}

\author{
Alasdair Bamford ${ }^{1,2} \cdot$ Garth Dixon $^{2,3} \cdot$ Nigel Klein $^{1,2} \cdot$ Stephen Marks ${ }^{2,4} \cdot$ Nicole Ritz $^{5,6} \cdot$ Steven B. Welch ${ }^{7}$ \\ Marc Tebruegge $^{2,6,8}$
}

Published online: 16 February 2021

(C) IPNA 2021

\section{Correction to: Pediatr Nephrol} https://doi.org/10.1007/s00467-020-04844-5

Unfortunately, the affiliation of the author group has been incorrectly published in original version. The complete correct affiliation of all authors should read as follows. The original version has been corrected.

Alasdair Bamford 1,2 Garth Dixon 3,2 Nigel Klein 1,2 Stephen Marks 4, 2 Nicole Ritz 5,6 Steven B Welch 7. Marc Tebruegge 2,6,8

1. Department of Paediatric Infectious Diseases, Great Ormond Street Hospital for Children NHS Foundation Trust, London, UK.

2. University College London Great Ormond Street Institute of Child Health, NIHR Great Ormond Street Hospital Biomedical Research Centre, London, UK.

3. Department of Paediatric Microbiology, Great Ormond Street Hospital for Children NHS Foundation Trust, London, UK.

The online version of the original article can be found at https://oi.org/ 10.1007/s00467-020-04844-5

Alasdair Bamford

a.bamford@ucl.ac.uk

1 Department of Paediatric Infectious Diseases, Great Ormond Street Hospital for Children NHS Foundation Trust, London, UK

2 University College London Great Ormond Street Institute of Child Health, NIHR Great Ormond Street Hospital Biomedical Research Centre, London, UK

3 Department of Paediatric Microbiology, Great Ormond Street Hospital for Children NHS Foundation Trust, London, UK

4 Department of Paediatric Nephrology, Great Ormond Street Hospital for Children NHS Foundation Trust, London, UK

4. Department of Paediatric Nephrology, Great Ormond Street Hospital for Children NHS Foundation Trust, London, UK.

5. University of Basel Children's Hospital, Paediatric Infectious Diseases and Vaccinology Department, Migrant Health Service, Basel, Switzerland.

6. Department of Paediatrics, Royal Children's Hospital Melbourne, University of Melbourne, Melbourne, Australia.

7. Birmingham Chest Clinic and Heartlands Hospital, University Hospitals Birmingham, Birmingham, UK.

8. Department of Paediatric Infectious Diseases and Immunology, Evelina London Children's Hospital, Guy's and St Tomas' NHS Foundation Trust, London, UK.

Publisher's note Springer Nature remains neutral with regard to jurisdictional claims in published maps and institutional affiliations.

5 Paediatric Infectious Diseases and Vaccinology Department, Migrant Health Service, University of Basel Children's Hospital, Basel, Switzerland

6 Department of Paediatrics, Royal Children's Hospital Melbourne, University of Melbourne, Melbourne, Australia

7 Birmingham Chest Clinic and Heartlands Hospital, University Hospitals Birmingham, Birmingham, UK

8 Department of Paediatric Infectious Diseases and Immunology, Evelina London Children's Hospital, Guy's and St Tomas' NHS Foundation Trust, London, UK 